\title{
On Fuzzy inference system based Failure Mode and Effect Analysis (FMEA) methodology
}

\author{
Kai Meng Tay \\ Electronic Engineering Department, Faculty of Engineering, \\ University Malaysia Sarawak \\ Sarawak, Malaysia \\ kmtay@feng.unimas.my
}

\begin{abstract}
Failure Mode and Effect Analysis (FMEA) is a popular problem prevention methodology. It utilizes a Risk Priority Number (RPN) model to evaluate the risk associated to each failure mode. The conventional RPN model is simple, but, its accuracy is argued. A fuzzy RPN model is proposed as an alternative to the conventional RPN. The fuzzy RPN model allows the relation between the RPN score and Severity, Occurrence and Detect ratings to be of non-linear relationship, and it maybe a more realistic representation. In this paper, the efficiency of the fuzzy RPN model in order to allow valid and meaningful comparisons among different failure modes in FMEA to be made is investigated. It is suggested that the fuzzy RPN should be subjected to certain theoretical properties of a length function e.g. monotonicity, sub-additivity and etc. In this paper, focus is on the monotonicity property. The monotonicity property for the fuzzy RPN is firstly defined, and a sufficient condition for a FIS to be monotone is applied to the fuzzy RPN model. This is an easy and reliable guideline to construct the fuzzy RPN in practice. Case studies relating to semiconductor industry are then presented.
\end{abstract}

Keywords- Fuzzy inference system, monotonicity property, sufficient conditions, FMEA, manufacturing

\section{INTRODUCTION}

Failure Mode and Effect Analysis (FMEA) is an effective problem prevention methodology that can easily interface with many engineering and reliability methods [1]. It can be described as a systemized group of activities intended to recognize and to evaluate the potential failures of a product/process and its effects [2]. Besides, FMEA identifies actions which can eliminate or reduce the chances of potential failures from recurring. It also helps users to identify the key design or process characteristics that require special controls for manufacturing, and to highlight areas for improvement in characteristic control or performance [1].

Conventional FMEA use a Risk Priority Number (RPN) to evaluate the risk associated to each failure mode. A RPN is a product of the risk factors, i.e., Severity (S), Occurrence (O) and Detect (D). FMEA assumes that multiple failure modes exist, and each failure mode has a different risk level that have to be evaluated, and ranked. In general, S, O and D are of integer 1 to 10 , usually defined in scale tables.

From literature, the use of Fuzzy Inference System (FIS) in FMEA is not new. Bowles and Peláez suggest to replace the conventional RPN model with a FIS (fuzzy RPN model) [3]. The fuzzy RPN model allows the relationship between the RPN score and the three risk factors (S, O, and D) to be of a non-linear relationship, which is too complicated to be modeled by the simple conventional RPN model. Motivation of FIS to be chosen in this problem domain can explained with FIS's ability to incorporate human/expert knowledge, whereby information is described by vague and imprecise statements $[4,5]$. Over the years, FIS models have demonstrated good capability and performances in a variety of application domains, e.g. control, modeling, and classification problems [4, 5].

The fuzzy RPN model is a popular method, and has been successfully applied to a number of FMEA problems. For example, it was applied to FMEA of an auxiliary feed water system and a chemical volume control system in a nuclear power plant $[6,7]$. It was also used in FMEA of an engine system [8], a semiconductor manufacturing line [9], and a fishing vessel [10]. Over the years, several enhancements have also been proposed to the fuzzy RPN model. Development of a fuzzy RPN model using the grey relation theory is presented in [10]. In [8], a fuzzy RPN model which allows interdependencies among all failures to be considered is proposed. In [9], a method to reduce the number of fuzzy rules in the fuzzy RPN model is reported.

However, little attention is paid on the validity and the efficiency of the estimated RPN scores, as available in the literature. Therefore, in this paper, the efficiency of the fuzzy RPN model, in order to allow valid and meaningful comparisons among different failure modes in FMEA to be made is investigated. The fuzzy RPN model is viewed as an assessment or measurement model, which is subjected to some theoretical properties of a length function, e.g. monotonicity, sub-additivity and etc [11].

Investigation in this paper focuses on monotonicity property of the fuzzy RPN model. The fuzzy RPN model is firstly presented. Monotonicity property in FIS and a sufficient condition for a FIS to be monotone is also reviewed. Monotonicity property for the fuzzy RPN model is further defined. In this piece of work, the sufficient conditions for a FIS to be monotone, as pointed in various sources $[12,13,14,15]$, is applied to the fuzzy RPN model. The sufficient conditions pointed out that for a FIS to be of monotonic, two mathematical conditions are needed. Condition (1) can be viewed as a method how membership function should be tuned in order to ensure the fuzzy RPN to be of monotonicity property. Condition (2) highlights the important of having a monotonic rule base in the fuzzy RPN 